\title{
Research review of transformer vibration monitoring technique
}

\author{
Yunze Yang ${ }^{1}$, Chang Wang ${ }^{1 *}$, Shujuan $\mathrm{Li}^{1}$, Li Min ${ }^{1}$ and Yuanyuan Yang ${ }^{1}$ \\ ${ }^{1}$ Laser Institute, Qilu University of Technology (Shandong Academy of Sciences), Jinan, Shandong, 250103, China
}

\begin{abstract}
Transformer vibration monitoring technique has been known to be safe, stable and resistant to interference. Based on the theories of transformer vibration and its origins, this study focuses on the equivalent mathematical model of transformer vibration, signal characteristics, as well as monitoring system for transformer vibration, to summarize research findings over recent years. A series of intelligent technologies have been adopted to reach certain diagnoses and monitoring outcomes; however, their applications are to some extent limited by the experience of users and other variants. The stability and reliability of techniques for monitoring transformer vibration need further improving.
\end{abstract}

\section{Introduction}

Transformer is one of the most critical equipment in the power system. According to incomplete statistics, its total energy consumption accounts for about $40 \%$ of the entire power system1. In the process of continuous operation for a long time, the power transformer is affected by the external environment, load increase, component aging, later maintenance, and other factors. Its insulation performance, internal circuit performance, mechanical performance and operating performance will gradually decline and result in malfunctions 2 . In severe cases, it could even cause grid failures, leading to the collapse of power systems and huge economic loss. Many faults are not discovered in time after they appear, which leads to further increase in their severity. The preprevention and diagnosis cycle are too long to accurately monitor the further development of the fault, so it is very necessary to find and deal with the fault in real time. The mechanical stability of the transformer winding is the most important factor affecting the short-circuit resistance of the transformer3. Studies have shown that in the transformer fault statistics, its windings, iron cores and on-load tap changers (OLTC) account for a very large proportion, of which winding and iron core faults account for about $70 \%$ of the total faults 4 .

The connection between the sensor and the transformer is mostly magnetic adsorption, so there is no electrical connection between the signal acquisition equipment and the transformer, which has no effect on the safe operation of the entire electrical system56. In the use of vibration analysis method, signal processing technology is particularly important. The technology is to use appropriate methods to transform the detected initial signals, and extract the features of useful signals from different angles. The key core of the technology is the selection of processing methods, the extraction and expression of feature information, and the accuracy of the results. The above factors have a greater impact on the completion of the fault diagnosis system 7 .

\section{Mechanism and propagation characteristics of transformer vibration}

Kang, P8 et al. found that during the operation of the transformer, the vibration frequency of the cooling device is usually concentrated below $100 \mathrm{~Hz}$, and the transient vibration frequency of the on-load tap-changer during normal operation is usually several kilohertz. In the process, the vibration of the winding and the iron core exists from beginning to end. The fundamental frequency of the vibration is $100 \mathrm{~Hz}$, which is twice the power frequency. In addition, due to the influence of the non-linearity of the iron core magnetostriction, there is also a fundamental frequency. The doubling frequency component and high-order harmonic components.

The vibration generated by the iron core, windings and cooling system needs to be transmitted to the surface of the transformer tank through different paths. The transmission path is shown in Fig.1.

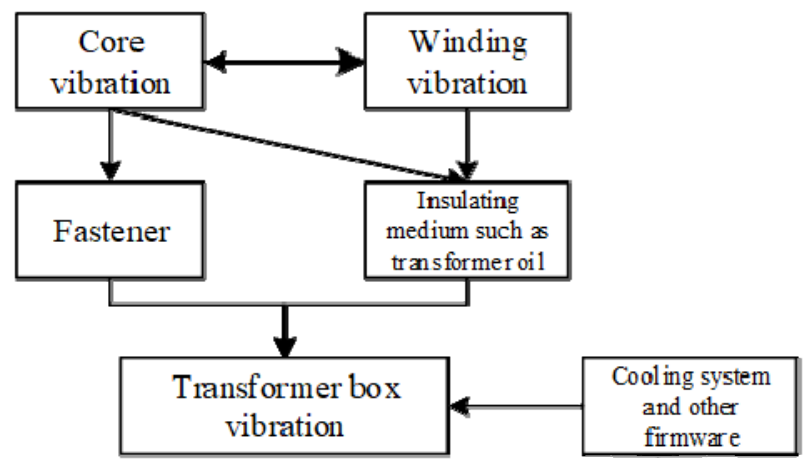

Fig.1. Transformer vibration source and transmission route. 9

\footnotetext{
*Corresponding author: yangyunze1024@126.com, wangchang@sdas.org
} 


\section{Research on modeling of transformer vibration}

Over the years, based on the concept of "system", some researchers have established models of the transformer as a whole or its windings and core (main vibration source) and other components, and tried to use the model to analyze, describe and reconstruct the normal operation of the transformer and its main components. Under the vibration state, in order to further study the transformer vibration monitoring and fault diagnosis later.

\subsection{Mathematical model of the establishment of transformer vibration}

Captions should be typed in 9-point Times. They should be centred above the tables and flush left beneath the figures.

\subsubsection{Modeling of winding vibration}

The windings of the transformer are made of multilayer wire cakes, and there are insulating pads between the multilayer wire cakes. The windings are immersed in transformer oil, and the upper and lower ends are clamped and fixed by the firmware. When the multilayer wire cakes are electrically driven When it is applied, the winding will vibrate. This vibration is the result of the interaction of the multilayer wire cake, insulating pad, insulating oil and firmware. Through the above theoretical explanation and experimental analysis of the overall mechanical characteristics of the winding, the classic spring-mass model is proposed. The vibration model is shown in Fig.2.

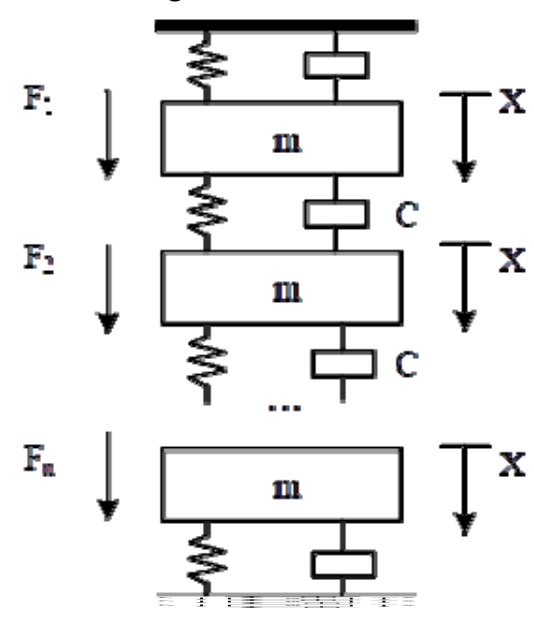

Fig.2. Axial vibration model of transformer winding.10

Starting from the transformer winding vibration model, using a series of mathematical methods through the law of motion equations, formula (1) is derived, which is the mathematical expression of the vibration model.

$$
M \frac{d^{2} x}{d t^{2}}+C \frac{d x}{d t}+K x=F+M g
$$

In the formula, $\mathrm{F}$-electromagnetic force ( $\left.F=\sum_{i=1}^{n} F_{n}\right) ; \mathrm{M}$-mass of the pancake coil $(\mathrm{M}=\mathrm{nm})$; $\mathrm{C}$ - damping coefficient; $\mathrm{K}$ - stiffness coefficient; $\mathrm{x}$ axial displacement of the pancake coil.

Swihart11-12 first proposed the above-mentioned classic spring-mass vibration model. According to this model, the axial vibration (displacement, velocity, acceleration, etc.) of the transformer winding can be specifically analyzed.

Based on the proposal of the spring mass model in classical mechanics, the winding wire cake is equivalent to the mass model, and the calculation is carried out using the multi-degree-of-freedom vibration method13. Among them, Hori and Patel are important representatives for the vibration research of this model under transformer short-circuit impulse14-16. Pater, McNutt, and others also proposed a three-element model to describe the third-order nonlinear characteristics of winding vibration. In the later scientific research process of Hori17, the nonlinear process of winding vibration changing with electromagnetic force was analyzed in more detail. After that, many scholars fitted the strain and stress relationship formulas of winding spacers under different conditions to objectively express Its nonlinear characteristics. The domestic research on winding vibration is relatively late. Chen Zhenmao18 and others first conducted the nonlinear study of the winding singledegree-of-freedom vibration model, and later analyzed the multi-dimensional model considering the constraint conditions, and Wang Hongfang19 also conducted related analysis. the study. The research ideas and results of scholars such as Li Hongkui and Shen Ming2021 are basically based on the winding vibration analysis of Hori et al.

\subsubsection{Model of iron core electromagnetic vibration}

The current electromagnetic-related theories have not yet fully explained magnetostriction, so some approximate models are often used to describe the magnetostriction behavior.

Based on the theory of magnetic field and elastic mechanics, Wang Zhimin22 et al. selected monolithic silicon steel sheet from the transformer core as the experimental object. They detailed the process of establishing mathematical model of core vibration, and the characterization of magnetostriction from the perspective of energy conservation and function conversion. Phenomenon of the magnetic field force, and derived a mathematical model of the electromagnetic vibration of the transformer core. Hilgert23 and others established transformer core vibration models that do not include magnetostrictive effects and include magnetostrictive responses based on neural network algorithms, and used these two models to calculate the actual 50kVA transformer vibration and compare them with the measured values.

The following limitations and shortcomings were found in the above results: 
(1) The existing mathematical models of windings mostly focus on the axial vibration of the windings, and rarely involve the establishment of the radial vibration model of the windings. However, for the overall vibration of the transformer, the radial vibration of the winding is also one of the main vibration sources of the surface vibration of the transformer tank, and it also contains important information about the mechanical structure of the winding, which is an important research object that cannot be ignored.

(2) The establishment process of the abovementioned vibration models is mostly theoretical calculations, and most of them have not been tested in practice and lack practicality.

\subsubsection{Based on finite element modeling and simulation technology transformer vibration}

Xie Poan of Shanghai Jiaotong University and his research team 2427 established finite element parameter models for windings and cores, and studied the actual measured vibration frequency response curves of transformer faults and the results obtained through simulation calculations. The influence of vibration frequency response curve characteristics. In view of the above research content, there are the following precautions. The first is the database for vibration monitoring data. This information will be used as the "fingerprint" of the transformer. Second, the operating data (transformer load, temperature, fault current, etc.) can be foreseen and abnormal Occurrence will change the internal conditions or transformer performance. This change can be measured by comparing the "fingerprint" vibration data with the last measurement result. Fig. 3 is a schematic diagram of the vibration frequency response experiment.

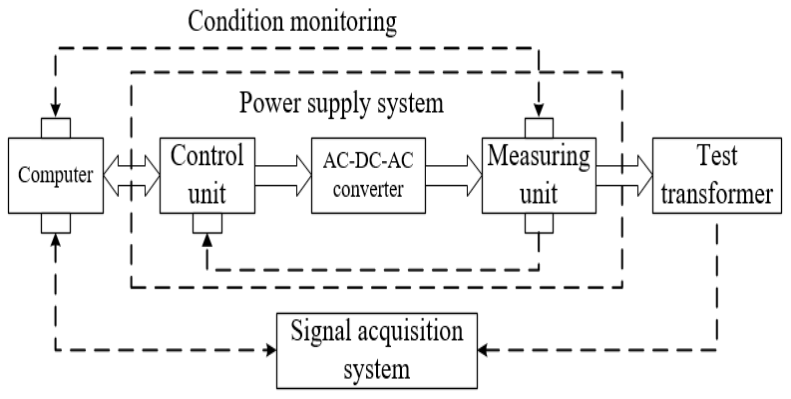

Fig.3. Schematic diagram of vibration frequency response experiment. 27

Li2829 et al. further studied the natural frequency change characteristics of windings under different compression forces through FEM models. Meng3031 and others used finite element analysis software to model high-voltage winding coils, and studied the relationship between winding force and deformation. Hackl A.32 et al. used a laser scanner to obtain the surface vibration velocity distribution map of the fuel tank and compare it with the FEM modeling results.

The finite element analysis method has high accuracy and wide application range. It has been widely used in the research of transformer vibration. However, there are still many problems in using the finite element FEM method to model the transformer vibration:

(1) When modeling the transformer, its internal structure is composed of many nonlinear materials and there are many nonlinear couplings, so it is difficult to obtain accurate physical parameters to establish a finite element model.

(2) In the simulation modeling of large power transformers, it is difficult, high load and slow, which leads to poor practicability.

\section{Transformer vibration characteristics and monitoring system}

\subsection{Research status of vibration signal characteristics of power transformers}

Through the characteristic analysis and extraction of the vibration signal on the surface of the transformer, the real-time working conditions of the winding and the iron core can be accurately reflected, and the operation status of the transformer can be monitored by the vibration analysis method.

\subsubsection{Current status of research on vibration characteristics of power transformer core}

Among foreign scholars, Japanese scholar Omura Takeshi33 used laser Doppler vibrometer, accelerometer and strain gauge to study the three-dimensional vibration and magnetostriction characteristics of silicon steel sheet as a transformer core. IEEE researcher Mohammed3435 of Florida International University and others established the analytical equation of the magnetostriction curve to calculate the unit magnetostriction force. The model uses the magnetization curve data to directly generate the magnetostriction curve $\lambda(\mathrm{H})$. Generated, based on the measurement of the inverse magnetostrictive effect, Fig. 4 is a schematic diagram of the measurement method.

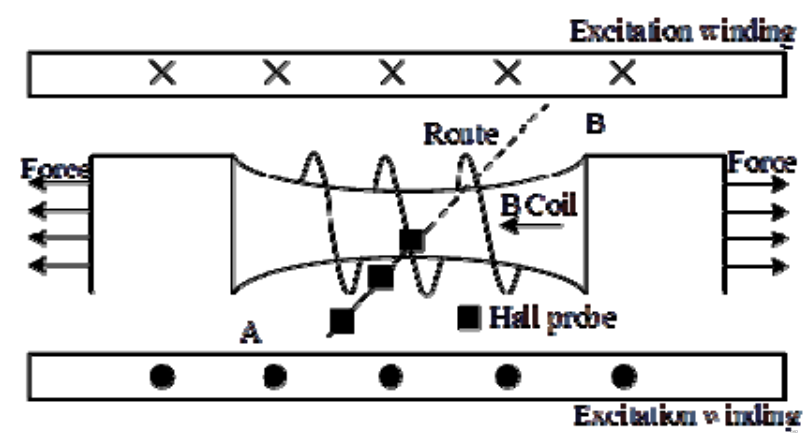

Fig.4. Method of measuring the inverse magnetostriction effects. 3435

\subsubsection{Research status of vibration characteristics of power transformer windings}

Naranpanawe Lalkshitha of the University of Queensland, Australia36 stablished a simplified threedimensional finite element model of $100 \mathrm{kVA}$, 
$10.1 / 0.4 \mathrm{kV}$ three-phase transformer windings based on the mass-spring block model and Ansys software in the vibration analysis of power transformer windings, as shown in Fig.5. As shown, a comparative experiment was carried out on the model parameters, and the influence of the mechanical factors of the winding on the winding vibration was studied.

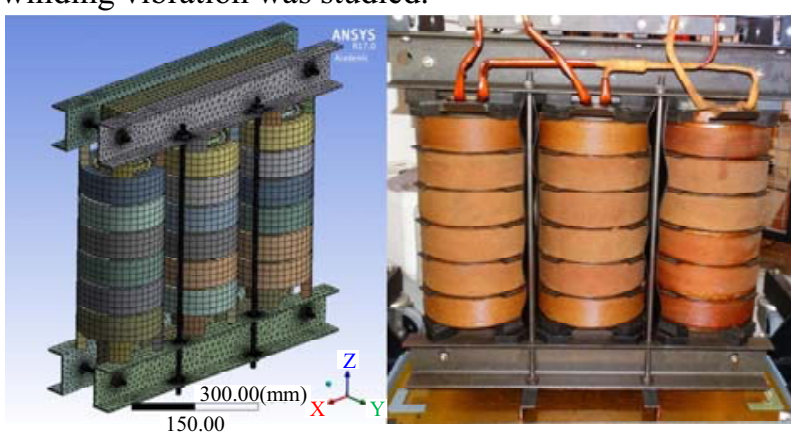

Fig.5. 10.1/0.4kV, 100kVA step-down distribution transformer (a) 3D model, (b) actual transformer.

Queensland University of Technology Hua-Shu Liu37 studied the influence of the thermodynamic stress of transformer windings on winding vibration through finite element simulation analysis, and developed a detailed FEA model to predict the dynamic response and thermal stress of the laboratory transformer, which is like the previous model. In contrast, this FEA model contains specific structural details. As shown in Fig.6, the thermal stress on the transformer increases with temperature, and the largest thermal stress occurs in the bolt part.

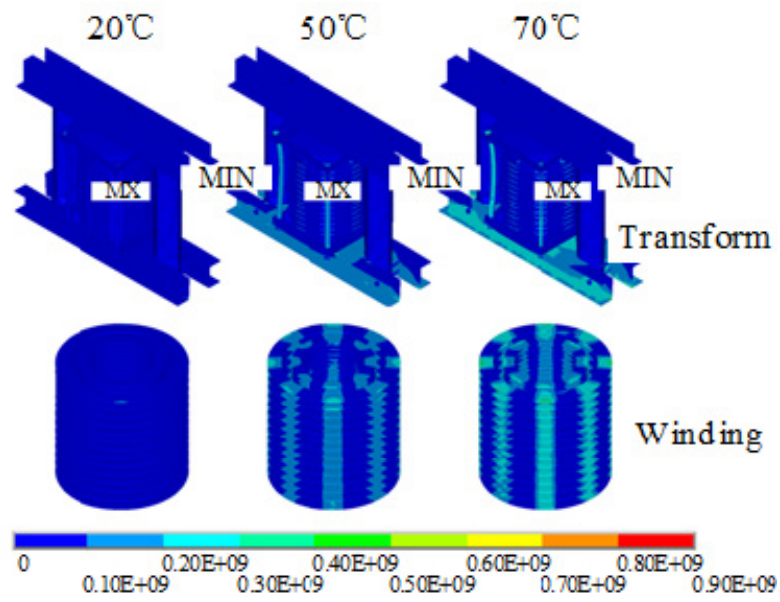

Fig.6.Thermal stress in the transformer during the temperature arising process. 37

Dr. Ji from Xi'an Jiaotong University 38 conducted a comprehensive simulation analysis on the axial vibration characteristics of a transformer with a model of SY50/10 under steady-state conditions. Dr. Cao39 from Shenyang University of Technology used more the physical field coupling software COMSOL Multiphysic takes a transformer of model S11-M-500/35 as the research object, and establishes the electric-magneticsolid three-field coupling model of the transformer under load conditions, which can better reflect the vibration information of the winding provides a theoretical basis for the selection of the measuring point position of the transformer body in the field test.

\subsection{Research status of transformer vibration monitoring system}

At present, the vibration monitoring systems developed in China are basically used for experimental analysis and research. The basic structure of the monitoring system 40 can be shown in Fig.7.

In foreign countries, Russia41 already has an online monitoring system used in more than 60 large power transformers, and the effect is good. The system developed by F. Poza42 is currently a relatively good online monitoring system in all aspects. Spain Carcia Belen43 of Carlos III University of Madrid, etc. studied on-line monitoring of power transformer winding deformation faults by vibration method, and proposed a power transformer tank surface vibration calculation model for power transformer online monitoring system.

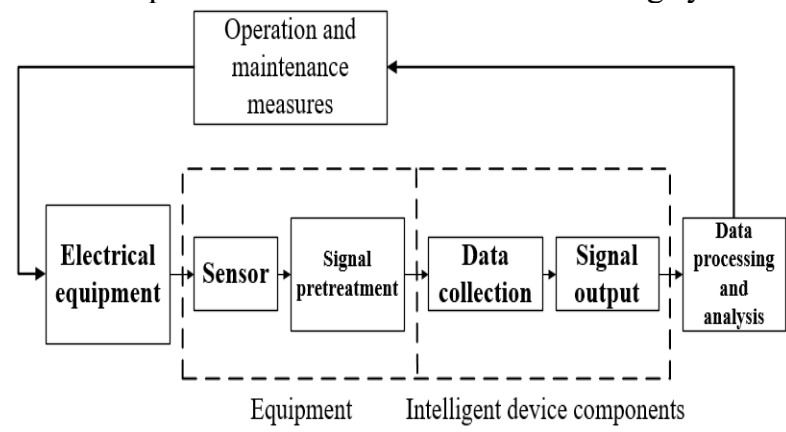

Fig.7. The basic structure of the monitoring system. 40

Yang Wenrui44 and other research and development an online monitoring system for transformer status based on vibration method is developed. The system uses vibration sensors, real-time controllers, and data acquisition cards to complete data collection. The upper computer software implements data analysis, data storage and data query functions through Labview. Wang Zebo45 and others designed and implemented a transformer fault diagnosis system based on the vibration method, and described the design of the software module in detail. The system integrates a variety of diagnostic algorithms to make timely alarms for abnormalities that occur during the operation of the transformer.

\section{Conclusions}

Issues that should be further studied in the field of transformer vibration monitoring.

(1) At present, most of the transformer vibration signal analysis methods can only qualitatively judge the state, and the universality and accuracy need to be improved.

(2) The current research mainly focuses on medium and small test transformers, and more attention should be paid to large transformers in the future.

(3) The normal operation of the transformer vibration monitoring system will obtain a large amount of vibration monitoring data. How to analyze the data and 
extract the characteristic information to achieve accurate state judgment is an urgent problem to be solved.

\section{Acknowledgments}

This article is one of the phased results of the National Key R\&D Program of China (2017YFB0405505), Shandong Province major scientific and technological innovation projects (2019JZZY010113), Science, Education and Industry Integration Project (2020KJCCG01) and Jinan innovation team project (2019GXRC028).

\section{References}

1. Gao, H.Y. (2019) Study on economic operation and optimal control strategy of transformer. Liaoning: Liaoning University of Technology.

2. Zhang, Y.P., Hu H.Y., Liu Q.Z., et al. (2018) Typical fault analysis and online monitoring technology for transformers. Safety Health \& Environment,09:11-14.

3. Zhao, Z.G., Li, G.F., Li, J.Z., et al. (2014) Analyzing the short-cinuit withstanding ability of large power transformer based the FEM method. High Voltage Engineering,40(10):3214-3220.

4. Li, X.L., Huang, H., Chen, X.X., et al. (2008) Design of on-line monitoring system for power transformer based on vibration analysis method. Transformer, 45(12): 60-64.

5. Chen, J., Ding Q.L., Li Z. (2011) Feasibility study of vibration monitoring for wave spectrum online transformer. Transformer, 48(9): 32-34

6. Dedus, A.F., Dedus, F.F., Makhortykh, S.A., et al. (1995) Generalized spectral-analytic method: part I theoretical foundations. Proceedings of SPIE-The International Society for Optical Engineering, 2363.

7. Huang, W.H. (1996) The principle, technology, and application of equipment fault diagnosis. Science Press, Beijing.

8. Kang, P., Birtwhistle, D. (2001) Condition monitoring of power transformer on-load tapchangers. Part 1: Automatic condition diagnostics.IEE Proceedings-Generation, Transmission and Distribution, 148(4): 301-306.

9. Gordy, T.D. (1950) Audible noise of power transformers. Electrical Engineering, 69(9): 800-805.

10. Ji, S.C., Wang, J.D., Li, Y.M. (2006) Research on axial vibration characteristics of transformer windings under steady condition. Advanced Technology of Electrical Engineering and Energy, 01: 35-38.

11. Swihart, D.O., Wright, D.V. (1976) Dynamic stiffness and damping of transformer pressboard during axial short circuit vibration. IEEE Transactions on Power Apparatus \& Systems, 95(2): 721-730.
12. Swihart, D.O., Mccormick, L.S. (1980) Short circuit vibration analysis of a shell form power transformer. IEEE Transactions on Power Apparatus Systems, 99(2): 800-810.

13. Uchiyama, N., Saito, S., Kashiwakura, M., et al. (2000) Axial vibration analysis of transformer windings with hysteresis of stress-and-strain characteristic of insulating materials. Power Engineering Society Summer Meeting, IEEE.

14. Watts, G.B. (1963) A mathematical treatment of the dynamic behaviour of a power-transformer winding under axial short-circuit forces. Proceedings of the Institution of Electrical Engineers, 110(3): 551-560.

15. Hiraishi, K., Hori, Y., Shida, S., et al. (1971) Mechanical strength of transformer windings under short-circuit conditions. IEEE Transactions on Power Apparatus Systems, 90(3): 2381-2390.

16. Patel, M.R. (1973) Dynamic response of power transformers under axial short circuit forces part Iwinding and clamp as individual components. Power Apparatus \& Systems IEEE Transactions on, PAS-92(5): 1558-1566.

17. Hori, Y., Okuyama, K. (1980) Axial vibration analysis of transformer windings under short circuit conditions. Power Apparatus \& Systems IEEE Transactions on, PAS-99(2): 443-451.

18. Chen, Z.M., Xu, J.X. (1990) Coil nonlinear vibration of large power transformer winding. Chinese Journal of Applied Mechanics, 01: 21-29.

19. Wang, H.F., Wang, N.Q., Li, T.S. (2000) Axial nonlinear vibration of large power transformer winding. Power System Technology, 03: 42-38.

20. Li, H.K., Li Y. (2010) Axial vibration modal analysis of transformer windings under different levels of pre-compression. Electric Machines and Control, 08: 98-101.

21. Shen, M., Yin, Y., Wu J.D., et al. (2014) Experimental investigating on on-line monitoring of winding deformation of power transformers. Transactions of China Electrotechnical Society, 29(011): 184-190.

22. Wang, Z.M., Gu, W.Y., Gu, X.A., et al. (2004) Mathematical model for electromagnetic vibration in large power transformer cores. Transformer, 41(006): 1-6.

23. Hilgert, T., Vandevelde, L., Melkebeek, J. (2008) Comparison of magnetostriction models for use in calculations of vibrations in magnetic cores. IEEE Transactions on Magnetics, 44(6): p874-877.

24. Xie, P.A. (2008) Study on application of vibration analysis to the condition monitoring of power transformers windings. Shanghai: Shanghai Jiao Tong University,

25. Wang, F.H., Xu, J., Jin, Z.J., et al. (2010) Experimental research of vibration sweep frequency response analysis to detect the winding deformation 
of power transformer. Transmission and Distribution Conference and Exposition, IEEE.

26. Wang, F.H., Jin, Z.J. (2011) Using the vibration frequency response analysis method to detect the winding deformation of power transformer. Power \& Energy Society General Meeting, IEEE.

27. Lu, Q.Y., Wang, F.H., Jin, Z.J. (2011) A variable frequency constant current power supply for vibration analysis method to detect the winding deformation of power transformer. Power \& Energy Society General Meeting, IEEE.

28. Li, Y., Zhou, W., Jing, Y., et al. (2011) Axial vibration analysis of power transformer active part under short-circuit. International Conference on Electrical Machines \& Systems, IEEE.

29. Li, H.K., Li, Y., Yu, X.H., et al. (2009) Axial vibrations modal analysis and computation of power transformer windings under different levels of precompression.2009 International Conference on Applied Superconductivity and Electromagnetic Devices, IEEE.

30. Meng, Z., Wang, Z. (2005) The analysis of mechanical strength of $\mathrm{HV}$ winding using finite element method. Part I Calculation of electromagnetic forces.39th International Universities Power Engineering Conference, 2004. UPEC 2004. IEEE.

31. Meng, Z., Wang, Z. (2005) The analysis of mechanical strength of $\mathrm{HV}$ winding using finite element method. Part II Mechanical stress and deformation analysis.39th International Universities Power Engineering Conference, 2004. UPEC 2004. IEEE.

32. Hackl, A., Hamberger, P. (2010) Investigation of surface velocity pattern of power transformers tanks.XIX International Conference on Electrical Machines, IEEE.

33. Omura, T., Yamaguchi, H., Ishigaki, Y., et al. (2015)3D Vibration analysis on three phase transformer cores. Ieej Transactions on Fundamentals \& Materials, 135(7):414-423.

34. Mohammed, O.A. (2001) Numerical prediction of magnetostrictive behavior in non-oriented electrical steel sheets. Southeastcon IEEE.

35. Mohammed, O.A., Liu, S., Abed, N. (2004) Study of the inverse magnetostriction effect on machine deformation. Southeastcon, IEEE.

36. Lakshitha, N., Chandima, E. (2016) Finite element modelling of a transformer winding for vibration analysis. Power Engineering Conference. IEEE.

37. Liu, H.S., Ma, L., Gu, Y.T., et al. (2014) Numerical investigation of mechanical and thermal dynamic properties of the industrial transformer. International Journal of Computational Methods, 11(supp01): 1344012.

38. Ji, S.C., Cheng, J., Li, Y.M. (2005) Research on vibration characteristics of windings and core of oil- filled transformer. Journal of Xi'an Jiao Tong University, 39(6): 616-619.

39. Li, H., Chen, J.B., Cao, C., et al. (2015) Simulation and test on vibration of power transformer windings based on multiple physics coupling method. Northeast Electric Power Technology,2:6-9.

40. Wang, C.Z. (2006) Online monitoring and fault diagnosis of electrical equipment. Tsinghua University Press, Beijing.

41. Rusov, A. (1998) Monitoring clamping forces in windings and cores of large power transformers using their vibration characteristics. Elektricheskie Stanzii, 6: 52.

42. Poza, F., Marino, P., Otero, S., et al. (2006) Programmable electronic instrument for condition monitoring of in-service power transformers. IEEE Transactions on Instrumentation \& Measurement, 55(2): 625-634.

43. Garcia, B., Burgos, J.C., Alonso, A.M. (2005) Transformer tank vibration modeling as a method of detecting winding deformations part I: theoretical foundation. IEEE Transactions on Power Delivery, 21(1): 157-163.

44. Yang, W.R., Dong, H.K., Yu, F.R., et al. (2016) On-line monitoring system for transformer vibration based on vibration method. Transducer and Microsystem Technology, 35(01): 93-95+99.

45. Wang, Z.B., Zhou, J.P., Liu, J.M., et al. (2014) Design of portable transformer vibration monitoring and fault diagnosis system[J]. Computer Engineering, 40(011): 292-296. 